\title{
Mitochondrial Genome Encoded Proteins Expression Disorder, the Possible Mechanism of the Heart Disease in Metabolic Syndrome
}

\author{
Ningning Zheng ${ }^{a}$ Dan Weib ${ }^{b}$ Bo Dai ${ }^{b}$ Lanyan Zheng ${ }^{c}$ Mingyi Zhao ${ }^{d} \quad N$ Xin $^{a}$ \\ Zhihong Chi ${ }^{a}$ Yiting Zhao ${ }^{e}$ Tingxian Ma ${ }^{a}$ Rabita Jahane ${ }^{f}$ Luning Sun ${ }^{a}$ \\ aDepartment of Pathophysiology, College of Basic Medical Science, China Medical University, \\ Shenyang, 'Department of Pathophysiology, Graduate School, China Medical University, Shenyang, \\ 'Department of Pathogen Biology, College of Basic Medical Science, China Medical University, \\ Shenyang, dDepartment of Clinical pharmacy, Shenyang Pharmaceutical University, Shenyang, ePET-CT \\ Center, Cancer Hospital Chinese Academy of Medical Sciences, Beijing, International Education School, \\ China Medical University, Shenyang, China
}

\section{Key Words}

Metabolic Syndrome - Heart Disease - Mitochondrion Encoded Protein - Mitochondrial Ribosomal Proteins

\begin{abstract}
Background/Aims: The direct consequence of metabolic syndrome (MS) is the increased morbidity and mortality caused by the heart disease. We tried to explain why the heart is more severely damaged during MS from the point of mitochondria, the center of cellular metabolism. Methods: 1 . The classic diet induced MS rat model was used to observe the morphological changes of mitochondria by transmission electron microscope (TEM); 2 . The expression of mitochondrial DNA (mt-DNA) encoded proteins was observed by immunohistochemistry and Western blot; 3. The expression of mitochondrial ribosomal proteins (MRPs) was observed by real-time PCR. Results: 1 . The mitochondrial volume increased but the number was normal in myocardial cells of the MS rats. But in the hepatocytes and skeletal muscle cells, the mitochondrial number decreased; 2.The mt-DNA encoded protein cytochrome $b$ increased significantly in heart but decreased in liver and the ATPase 6 increased in liver but decreased in heart of the MS rats; 3. The mRNA levels of MRPS23, MRPL27, MRPL45 and MRPL48 elevated in heart but down-regulated in liver of the MS rats. Conclusion: The morphologic and functional alterations of mitochondrion in MS were tissue specific. Heart displays a distinctive pattern of mitochondrial metabolic status compared with other tissues.

(C) 2017 The Author(s)

Published by S. Karger AG, Basel
\end{abstract}




\section{Cellular Physiology Cell Physiol Biochem 2017;43:959-968 \\ \begin{tabular}{ll|l} 
and Biochemistry & $\begin{array}{l}\text { DOI: 10.1159/000481649 } \\
\text { Published online: September 29, } 2017\end{array}$ & $\begin{array}{l}\text { C } 2017 \text { The Author(s). Published by S. Karger AG, Basel } \\
\text { www.karger.com/cpb }\end{array}$ \\
\hline
\end{tabular}}

\section{Introduction}

Metabolic syndrome (MS) is a multi-factorial disorder with the clustering of several metabolic abnormalities such as obesity, dyslipidemia, hypertension, increased plasma glucose levels, prothrombotic condition, and proinflammatory condition occurring in the same individual, which leads to a major cardiovascular risk factor. Prevalence of MS in the United States ranged between $34.3 \%$ and $38.5 \%$ depending on the criteria that was employed [1]. It increases with age and tends to peak among those aged 60 69 years [2]. Prevalence of MS in China is lower, which is $13.7 \%$ [3]. In recent years, MS has increased very rapidly and aroused major concerns as one of the most alarming noninfectious diseases at the moment. Insulin resistance and central obesity are considered to be the evident pathogenic factors of MS [4, 5]. Other factors such as heredity, lack of physical activity, aging and inflammation are also identified to be relevant factors [6]. But the existing evidence is not strong enough to further clarify the underlying mechanisms unless the common ground of multiple metabolic disturbances in MS is found. It is well established that mitochondrion is the center of cell metabolism where the final steps of the oxidation of carbohydrate, lipid and protein complete through tricarboxylic acid cycle, and it is also the main source of reactive oxygen species (ROS). Therefore, mitochondrial dysfunction might induce increased oxidative stress and promote the progression of MS [7]. Mitochondrion is the only cellular organelle that contains DNA, and the proteins encoded by the mitochondrial genome are all subunits of respiratory chain complex, which suggested that these mitochondrial gene encoded proteins play crucial roles in cellular metabolism. To the present, the molecular biological alterations in mitochondrial genome have been proven to be the important mechanisms of MS [8-10]. The main components of MS, particularly visceral obesity, insulin resistance, and type 2 diabetes mellitus (T2DM) are all associated with changes in mitochondrial metabolism in different tissues. However, it is shown that the energy metabolism in different tissues is not the same and different organs are not equally affected in MS. As mentioned, the direct consequence of MS is the increased morbidity and mortality of heart disease. Therefore, one of the aspects that deserve particular consideration is the causative link between altered mitochondrial genome expression and the structural damage and dysfunction in different tissues, especially the characteristic change of heart. This might be a very important factor to further clarify the mechanisms and provide support for developing a treatment for MS heart disease.

In this study, we adopted the well established method of diet induced MS model to observe and compare the mitochondrial morphologic changes, the expression of mitochondrial genome encoded proteins and mitochondrial ribosomal proteins (MRPs) in liver, heart and skeletal muscle. Furthermore, we discuss the mitochondrial mechanisms of MS and try to find some solutions to the heart disease of MS according to the metabolic status of different tissues.

\section{Materials and Methods}

This animal study was approved by the Institutional Animal Care and Use Committee. All procedures in this study were carried out in accordance with the recommendations in the guide for the care and use of laboratory animals of China Medical University. All surgery was performed under anesthesia, and all efforts were made to minimize pain and suffering for the animals.

\section{Animals}

The diet induced MS rat model was used. 8-wk-oldmale Sprague-Dawley rats weighing 100 to $110 \mathrm{~g}$ (Experimental animal center of China Medical University) were kept in cage at $21 \sim 22^{\circ} \mathrm{C}$ on a $12 \mathrm{~h}$ : $12 \mathrm{~h}$ light dark cycle. 40 rats were classified into two groups randomly. 20 rats (controls) were given a diet of standard laboratory chow. The remaining 20 rats were fed with a high energy (HE) diet consisting of 20\% lard, $10 \%$ sugar, $10 \%$ dry milk solution, 2\%cholesteral, 0.5\%cholate, 5\%salt, 52.5\%standard laboratory chow for 16 week. Food intake and body weight were measured daily and water was continually available. 


\section{Cellular Physiology Cell Physiol Biochem 2017;43:959-968 \begin{tabular}{l|l|l} 
and Biochemistry & Published online: September 29, 2017 & $\begin{array}{l}\text { (c) } 2017 \text { The Author(s). Published by S. Karger AG, Basel } \\
\text { www.karger.com/cpb }\end{array}$
\end{tabular}

\section{Biochemical assays and sample preparation}

Whole blood was collected from the tail vein of the rats at the end of the 16th week in the fasting state, and the serum was collected by centrifugation at $3000 \mathrm{rpm} / \mathrm{minute}$ for 15 minutes. Fasting blood glucose(FBG), serum total cholesterol(TC), triglyceride(TG), low-density lipoprotein (LDL) and highdensity lipoprotein(HDL) were measured using the enzymatic colorimetric kits (BHKT clinical reagent Co. Ltd, Beijing China). The blood pressure was also measured in the resting state. Then all rats were anesthetized using $10 \%$ chloralhydrate $(300 \mathrm{mg} / \mathrm{kg}$, i.p.) and killed by decapitation. Liver, heart and leg muscles were removed and quartered. One quarter was immediately frozen on dry ice, and then stored at $80^{\circ} \mathrm{C}$ for protein and RNA extraction. One quarter was stored in formalin for immunohistochemistry (IHC). One quarter was cut into $1 \mathrm{~mm}^{3}$ cubes and fixed in the $2.5 \%$ glutaraldehydefor electron microscopy observation. The remaining one quarter was stored at $80^{\circ} \mathrm{C}$ as a backup.

Mitochondrial morphologic change

Transmission electron microscopy (TEM) was used to evaluate mitochondrial morphologic changes. The tissue mass of $1 \mathrm{~mm}^{3}$ size was post-fixed in $2.5 \%$ glutaraldehydein $0.1 \mathrm{M}$ phosphate-buffered saline (PBS), pH 7.4, at $4^{\circ} \mathrm{C}$ for $24 \mathrm{~h}$, and then further fixed in $1 \%$ osmiumtetroxide. Tissues were then processed through graded ethanol and embedded in Spurr's resin. Ultrathin sections were cut and floated onto 200 mesh copper grids (Agar Scientific Ltd. UK), and counter-stained with uranylacetate and lead citrate. Sections were viewed and photographed using a Hitachi H-7650 transmission electron microscope (Hitachi Company).

\section{Immunohistochemistry for mitochondrial genome encoded proteins}

A standardized protocol was used to analyze mitochondrial genome encoded proteins. Immunohistochemistry was performed as described [11]. Primary antibody of cytochrome b was diluted as 1:2000 and ATPase 6 was 1:500. Antibodies were purchased from Abcam, U.S.A. Slides were incubated with Vectastain Elite ABC (Vector Laboratories) and detected with DAB (Vector Laboratories).

Western blot for mitochondrial genome encoded proteins

Western blot was performed as described [12]. All the primary antibodies were purchased from Abcam and diluted as followed: NADH dehydrogenase 1 (ND1), 1:1000; cytochrome b, 1:2000; cytochrome c oxidase 2 (COX2), 1:500; ATPase 6, 1:500. HRP-labeled anti-rabbit and anti-mouse secondary antibodies were obtained from Santa Cruz Biotechnology.

\section{SYBR green based real time PCR for MRP}

Total RNA was extracted from frozen tissues using Trizol reagent (Invitrogen) and cDNA was synthesized by reverse transcription using random primers following the protocol recommended by Promega. MRP mRNA was detected using ABI Prism 7500 Sequence Detection System (Applied Biosystems). $\beta$-actin was used as an internal control. The primers were listed in Table 1. Each sample was amplified in triplicate to obtain average Ct value. Reactions without cDNA templates were used as negative controls. Results were expressed as relative MRP mRNA levels compared with the untreated control, which was considered as $100 \%$. All realtime PCR experiments were performed with 3 biological replicates in triplicates for each sample and standardized to $\beta$-actin expression levels.

\section{Statistical Analysis}

The data obtained was represented as mean \pm SD of at least three independent preparations. Statistical analysis was performed by the one-way analysis of variance (ANOVA) with $\mathrm{T}$ test. A difference with $P<0.05$ was considered statistically significant.
Table 1. PCR primer sequences and amplified length

\begin{tabular}{ccc}
\hline Gene & \multicolumn{1}{c}{ Primer Sequence } & Product length (bp) \\
\hline MRPL27 & F: 5'- CTGTCAGATCCGCATCCAAGAA-3' & 118 \\
& R: 5'- ATGTTGCCGGCATGAACGTA-3' & \\
MRPL45 & F: 5'-TCAGACCATGACCGGCTTCATA-3' & 88 \\
& R: 5'-TCCAGCGCACAGTCTTGTACTTG-3' & \multirow{2}{*}{149} \\
MRPL48 & F: 5'- TATGTCCACCGCCTCTGCAA -3' & \multirow{2}{*}{84} \\
& R: 5'- TGCACCACTCGTTCATGGGTA-3' & \multirow{2}{*}{ MRPS23 } \\
& F: 5'- GCCAAAGCTGACATCCAGGAC-3' & 81 \\
& R: 5'- TTTGTGACCAGATCCATAGGTTGAA-3' & \\
& F: 5'-AGCCATGTACGTAGCCATCCA-3' & \\
& R: 5'-TCTCCGGAGTCCATCACAATG-3' &
\end{tabular}




\section{Results}

The variations of biochemical criteria of MS rats

The diet induced MS animal models were obtained according to the standard protocol [13]. We compared the variations biochemical criteria in the serum between MS rats and the control group. The biochemical parameters of FBG, TC, TG, LDL and HDL were observed.

As expected, the concentration of FBG, TC, TG and LDL were higher and HDL was lower in MS rats than that of the control group $(P<0.05)$ (Fig. 1).

Mitochondrial morphologic change of MS rats

Electron microscopy revealed markedly reduced mitochondrial cristae density in the myocardial cells of MS rats but not in that of the control group. Although mitochondrial volume was increased in the myocardial cells of MS rats, mitochondrial number was unchanged between the groups (Fig. 2, Table 2). The mitochondrial ultra structure injury in the hepatocytes, skeletal muscle cells were accurate in measurements with the data collected from other literature and experiments [14]. In these cells, the mitochondrial numbers deceased, the mitochondrial cristae were broken and the matrix clearing was visible (Table 2).

\section{Expression of mitochondrial} genome encoded proteins

We examined the expression of mitochondrial genome encoded proteins cytochrome $b$, ATPase 6, ND1 and COX2 in different tissues of wild type and MS rat by Western blot analysis.

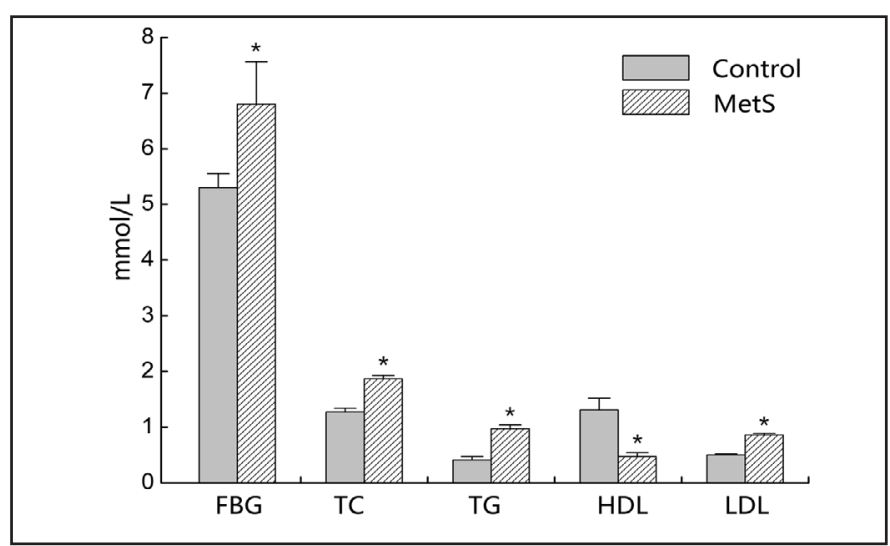

Fig. 1. The variations of biochemical criteria of MS rats. The concentration of FBG, TC, TG and LDL were higher and HDL was lower in MS rats compared with control $(\mathrm{P}<0.05)$.

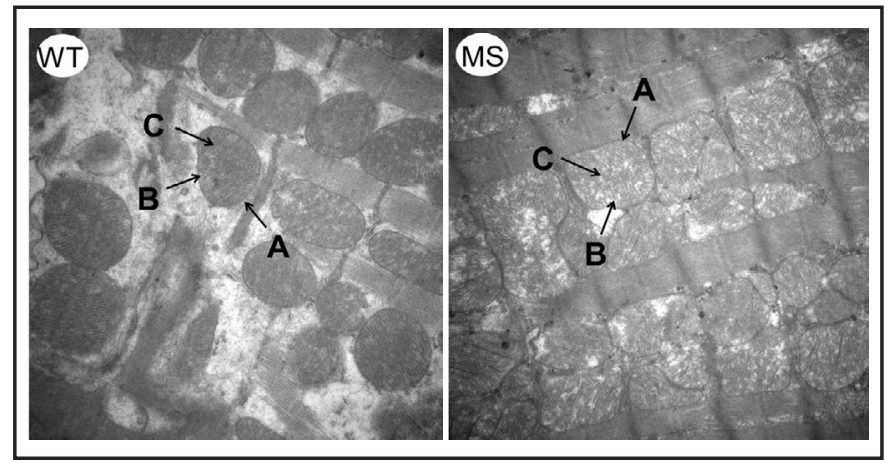

Fig. 2. The mitochondrial morphologic change in the cardiomyocyte of MS rat. The density of mitochondrial cristae was reduced significantly, mitochondrial volume was bigger and the number of mitochondria was unchanged in the cardiomyocyte of the MS rats compared with those of the control group. The original magnification: $\times 25000$. Scale bar $=1 \mu \mathrm{m}($ A: Mitochondria. B: Mitochondrial matrix. C: Mitochondrial cristae). Cytochrome $\mathrm{b}$ is a component

of respiratory chain complex III which catalyzes the reduction of cytochrome $\mathrm{c}$ by oxidation of coenzyme $Q(\mathrm{CoQ})$ and the concomitant pumping of 4 protons from the mitochondrial matrix to the intermembrane space. A significant amount of superoxide is produced at the level of complex I and II of the ETC [15]. The results showed that the expression of cytochrome $b$ increased significantly in heart and
Table 2. The number of mitochondria in different tissues of MS rat. ${ }^{*} \mathrm{P}<0.05$, compared with control group in liver; $\# \mathrm{P}<0.05$, compared with control group in muscle

\begin{tabular}{lcr}
\hline Tissue & Control & \multicolumn{1}{c}{ MS } \\
\hline Heart & $18.67 \pm 1.24$ & $17.33 \pm 0.47$ \\
Liver & $13.66 \pm 0.47$ & $9.67 \pm 1.70^{*}$ \\
Muscle & $14.33 \pm 1.25$ & $11.33 \pm 0.94^{\#}$ \\
\hline
\end{tabular}




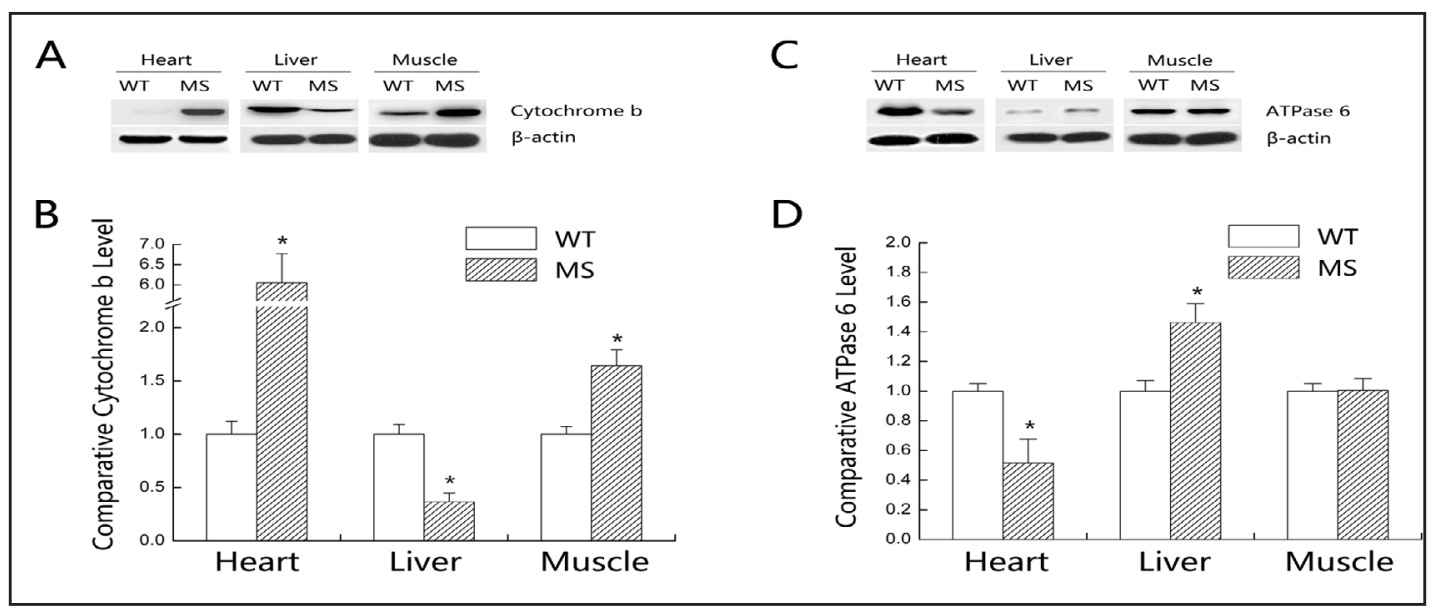

Fig. 3. Western blot analysis of wild type and MS rats for cytochrome b and ATPase 6 in heart, liver and muscle. A, B: Cytochrome b expression increased in heart and muscle and decreased in liver with $\beta$-actin as protein loading control. C, D: ATPase 6 expression increased in liver and decreased in heart, and no obvious change of ATPase 6 expression in muscle with $\beta$-actin as protein loading control.

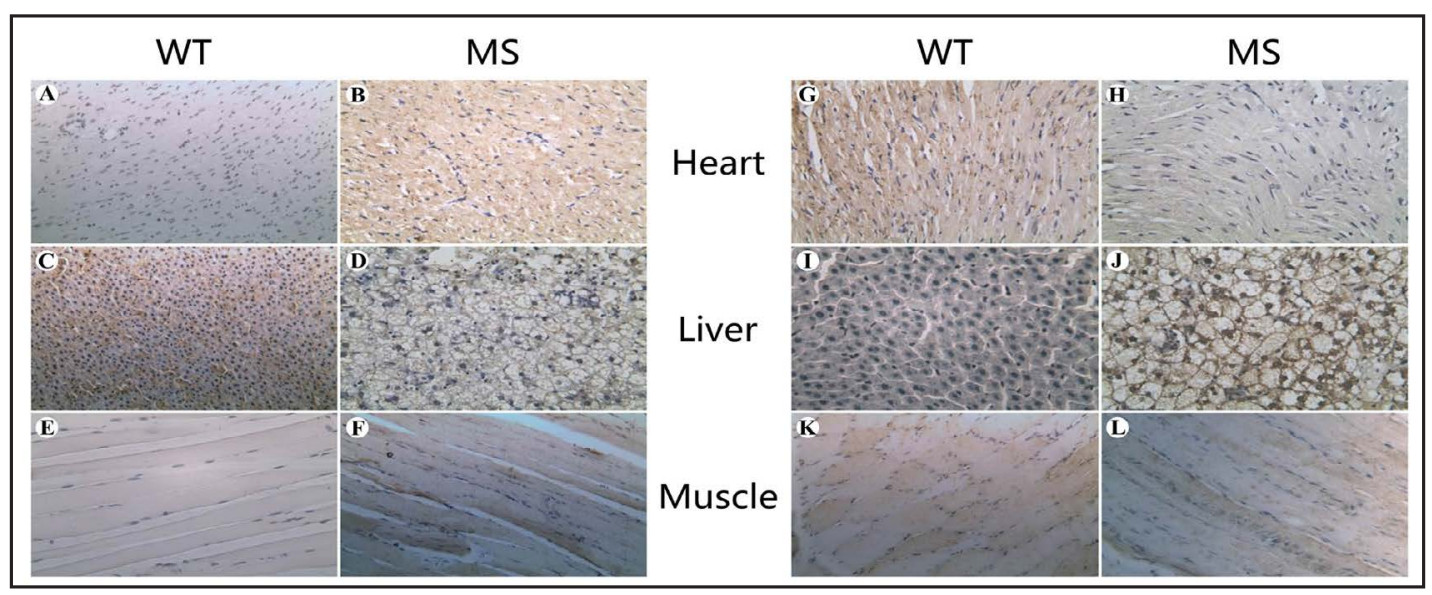

Fig. 4. Immunohistochemistry analyses of cytochrome $b$ and ATPase 6 in heart, liver and muscle of wild type and MS rats. A. Cytochrome b immunostaining in heart of wild type rats. B. Cytochrome b immunostaining in heart of MS rats. C. Cytochrome b immunostaining in liver of wild type rats. D. Cytochrome $\mathrm{b}$ immunostaining in liver of MS rats. E. Cytochrome $\mathrm{b}$ immunostaining in muscle of wild type rats. F. Cytochrome b immunostaining in muscle of MS rats. G. ATPase 6 immunostaining in heart of wild type rats. H. ATPase 6 immunostaining in heart of MS rats. I. ATPase 6 immunostaining in liver of wild type rats. J. ATPase 6 immunostaining in liver of MS rats. K. ATPase 6 immunostaining in muscle of wild type rats. L. ATPase 6 immunostaining in muscle of MS rats. The original magnification: $\times 400$. Scale bar $=200 \mu \mathrm{m}$.

muscle but decreased in the liver of MS rat (Fig. 3A and 3B, Fig. 4A-4F). ATPase 6 is a subunit of complex $\mathrm{V}$ that uses the energy of a transmembrane proton gradient for adding an inorganic phosphate group to a molecule of adenosine diphosphate (ADP) to form a molecule of adenosine triphosphate (ATP). After comparing with the wild type rat tissues, ATPase 6 expression was increased in the liver while decreased in the heart and no obvious changes was seen in muscles of the MS rats (Fig. 3C and 3D, Fig. 4G-4L). ND1, the mitochondrial electron transport complex, is a flavoprotein and iron sulfur-containing oxidoreductase that catalyzes the oxidation of NADH to NAD. All the hydrophobic subunits of complex I including ND1 are encoded by the mitochondrial DNA. No significant changes of the ND1 expression

\section{KARGER}




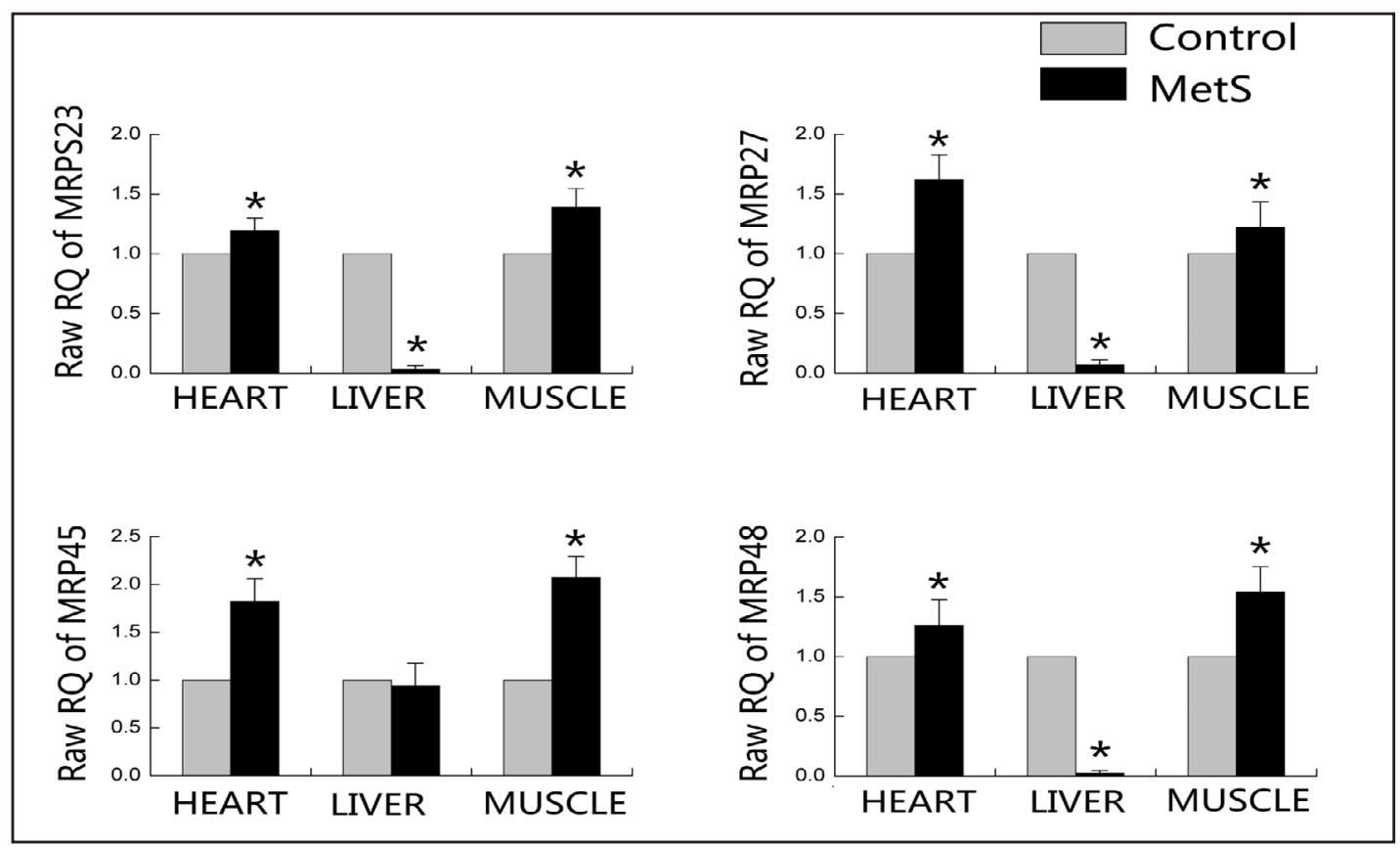

Fig. 5. A Real-time PCR analysis of wild type and MS rat for MRPS23, MRP27, MRP45 and MRP48.The expression of MRPS23, MRP27, MRP45 and MRP48 were all increased in heart and muscle, while MRPS23, MRP27 and MRP48 were decreased in liver in MS rat compared with control rat $(\mathrm{P}<0.05)$. There was no obviously difference about the expression of MRP45 between MS and control rat in liver (P>0.05). RQ: Relative Quantitation.

were found in different tissues of the MS rats (data not shown). The COX2 is the last enzyme in the respiratory electron transport chain of mitochondria, which receives an electron from each of four cytochrome c molecules, and transfers them to one oxygen molecule, converting molecular oxygen to two molecules of water. Similar to ND1, no significant changes of the COX2 expression were found in MS rats (data not shown).

\section{Expression of MRPs}

Mammalian mitochondrial ribosomal proteins (MRPs) are encoded by nuclear genes and help in protein synthesis within the mitochondrion, which may affect the synthesis of mitochondrial encoding proteins, and then, the efficiency of oxidative phosphorylation and the process of cell metabolism. Real time PCR analysis revealed elevated mRNA levels of MRPS23, MRPL27, MRPL45 and MRPL48 in heart and muscle, while significantly low levels of MRPS23, MRPL27 and MRPL48 in liver of the MS rats (Fig. 5).

\section{Discussion}

Mitochondria metabolizes nutrients to produce the "energy currency" ATP for normal cell function. The final stages of the metabolism of sugar, lipid and protein are all accomplished in the mitochondrion through the tricarboxylic. Therefore, the pathologic alterations of mitochondria contribute to the development of MS. Mitochondria are dynamic organelles that are continuously fusing and dividing. The balance of the two processes is responsible for the morphologic features and functions of mitochondria [16]. It was reported that the overexpression of fusion proteins, such as mitofusin 2 (Mfn2), results in the formation of large mitochondria, whereas the overexpression of fission proteins, such as the dynamin related protein 1 (Drp1) or mitochondrial fission protein 1(Fis1), results in the formation of small fragmented mitochondria. When fusion is decreased, as in OPA1 (optic 


\section{Cellular Physiology \\ \begin{tabular}{ll|l} 
and Biochemistry & $\begin{array}{l}\text { DOI: 10.1159/000481649 } \\
\text { Published online: September 29, } 2017\end{array}$ & $\begin{array}{l}\text { C } 2017 \text { The Author(s). Published by S. Karger AG, Basel } \\
\text { www.karger.com/cpb }\end{array}$ \\
\hline
\end{tabular}}

atrophy protein 1)-depleted cells, reduced glucose oxidation, decreased mitochondrial respiration, and diminished mitochondrial membrane potential will happen [17]. Indeed, each component of the MS also independently modulates mitochondrial morphology, function, proteome and biogenesis [18]. As mentioned, the energy metabolism in different tissues is not the same, and on the other hand, different tissues are not equally affected in MS. Mitochondrial morphologic changes in different tissues of MS rats have been observed in this study. Different from others, the volume of myocardiocytes increased significantly. Usually, mitochondrial size varies more in older cells, as compared to corresponding young cells, with a high proportion of large or sometimes giant mitochondria. The majority of normally autophagocytosed mitochondria are small, while larger mitochondria are less well autophagocytosed, and these damaged cellular structures might be considered as biological garbage. Moreover, mitochondrial enlargement can lead to the mitochondrial functional deficiencies such as decreased ATP production [19]. It is well established that heart has a greater demand for ATP than most other organs to maintain its normal function; therefore, the mitochondrial morphologic change of myocardiocytes might contribute to the progression of heart disease in MS. We know that the most direct consequence of the metabolic syndrome is the increase of heart disease morbidity and mortality [20]. Why the heart is more easily to be attacked in MS and what are the underlying mechanisms? The first factor, to consider is the feature of energy metabolism in myocardiocytes. It is well established that the metabolic way of the myocardiocytes is unique. Normally, fatty acids are the primary substrate of the adult heart; with $60 \% \sim 70 \%$ of the energy obtained from the oxidation of fatty acids, and the remainder come from the metabolism of carbohydrates which are the primary energy substrate of other cells. From the point of oxygen consumption, fatty acid ßoxidation need about 10\% more oxygen than glucose does to produce same amount of ATP. As a result, metabolic disorders are more easily to be found in the myocardiocytes during hypoxia because of their high demand of oxygen. At the same time, the heart is particularly susceptible to oxidative damage because more reactive oxygen species (ROS) will be generated. Therefore, switches in the myocardial substrate utilization and energy production rates have been shown to occur in various cardiomyopathies, as well as in any subsequent heart failure. Based on this question, we carried out relevant research and found that the expression of mitochondrial respiratory complexes in myocardial cells were significantly different from other cells. The mitochondrial electron transport chain (mETC), composed of four multi-subunit complexes (complex I-complex IV) and two electron carriers (CoQ and cytochrome c) generates a transmembrane proton gradient that drives ATP synthesis by complex V (ATP synthase) [21]. The fatty acid $\beta$-oxidation spiral involves four sequential enzymes: acyl-CoA dehydrogenase, 2-enoyl-CoAhydratase, L-3-hydroxyacyl-CoA dehydrogenase, and 3-ketoacyl-CoA thiolase [22]. Mitochondrial acyl-CoA dehydrogenases transfer single electrons to electron-transferring flavoprotein (ETF) [23], and the latter was oxidized by ETF-ubiquinone oxido-reductase (ETFQOR), which donates electrons directly to the ubiquinone pool in the mitochondrial inner membrane [24-26]. And then, electrons were passed to complex III of the respiratory chain, cytochrome c, complex IV, and finally molecular oxygen. Changes in mitochondrial complexes may have a large impact on cellular metabolism, which could be the core mechanisms of the heart diseases in MS. In this study, considering their crucial roles in cellular metabolism, we only examined the expression of mitochondrial genome encoded subunits of the mitochondrial respiratory chain complexes, i.e. ND1 in complex I, cytochrome b in complex III, COX2 in complex IV and ATPase 6 in complex V. The complex II, which is mainly coded by nuclear genes, was not involved in the experiment. Complex III is a central component of the respiratory chain, catalyzing transfer of electrons from ubiquinol to oxidized cytochrome c. It has been recognized that complex III is a key site for ROS generation in cardiac mitochondria and principle target of many cardiovascular risk factors [27, 28], and the ROS production is increased by fatty acids because of its partial inhibition of the electron transport [29-31]. However, it was observed that rat heart mitochondria oxidizing acylcarnitines of short-, medium- and long-chainfatty acids release only small amounts of ROS in the resting state [32]. Among the 11 subunits of 


\section{Cellular Physiology Cell Physiol Biochem 2017;43:959-968 \\ \begin{tabular}{ll|l} 
and Biochemistry & $\begin{array}{l}\text { DOI: 10.1159/000481649 } \\
\text { Published online: September 29, } 2017\end{array}$ & $\begin{array}{l}\text { C } 2017 \text { The Author(s). Published by S. Karger AG, Basel } \\
\text { www.karger.com/cpb }\end{array}$ \\
\hline
\end{tabular}}

complex III, cytochrome $b$ is the only one that is encoded by mtDNA, and its expression in the myocardiocytes of MS animals has not been published in the literature. Our data indicate that the elevated expression of cytochrome b might contribute to the development of the heart disease in MS. As to the underlying mechanisms, e.g. if the high expression of cytochrome $b$ could induce ROS generation in complex III when fatty acids are used as substrates in MS, still need further study.

ATPase is also known as mitochondrial respiratory chain complex V. It participates in the synthesis and hydrolysis of ATP. ATPase 6 is one of the two mitochondrial encoded subunits of ATPase (the other one is ATPase8) and it is a component of the proton channel, which is essential for proton transportation and energy production. Cell and mitochondrial function analysis indicated that some ATPase6 gene mutations led to energy deprivation, ROS overproduction and reduced ATP production [33-35]. A mitochondrial DNA mutationA8890G was reported in a Chinese patient with juvenile-onset metabolic syndrome [36]. However, there was no evidence that ATPase8 gene mutation is related to the etiology of MS. In the present study, we found that ATPase 6 expression decreased remarkably in the heart of the MS rats, by contrast, the expression increased in hepatocyte of the MS rats. Our result might partially explain why heart is usually more severely affected during MS.

The mRNAs for the mtDNA-encoded proteins are translated on mitochondrial ribosomes (mitoribosomes). Mitoribosomes can regulate gene expression by varying the efficiency of translation of mRNAs for OXPHOS [37]. It was reported that the alteration of certain mitochondrial ribosomal protein (MRP) may affect the expression of different mtDNAencoded proteins [38, 39]. Therefore, MRPs are the key components for mitochondrial biogenesis and the defects in MRPs might result in mitochondrial dysfunction and associate pathological conditions. It has been speculated that absence or deficiencies of MRPs may be a primary cause of some oxidative phosphorylation disorders such as systemic neuromuscular disorders, Leigh Syndrome and diabetes [40]. Besides that, MRPs also perform some specific roles, which are possibly distinct from those in the mitoribosome. For instance, MRPS38 has a role in the proteasome degradation pathway [41]; DAP3/MRPS29 is known to interact with proteins that contribute to cell apoptosis [42]. Certain MRP genes are present in candidate regions for human disorders. Therefore, it is worthwhile pursuing the possibility that defects in the MRP genes expression may result in such pathological conditions. Further attention should be given to the association between cardiac disease and the pathological condition mentioned since mitochondria play a vital role in the functioning of the heart by accounting for more than $90 \%$ of the ATP required for its functioning. Our real time PCR analysis revealed elevated mRNA levels of MRPS23, MRPL27, MRPL45 and MRPL48in heart and muscle, while significant low expression levels of MRPS23, MRPL27and MRPL48 in liver of the MS rats. The researches on the expression of these MRPs in human diseases at present are quite few. According to the few literatures in these years, MRPS23 was overexpressed in patients with highly proliferative luminal breast tumors [43] and MRPL48 was under-expressed in adrenocortical carcinoma (ACC) [44]. We found the expression change of the above MRPs in MS, more important; they exhibited apparent tissue specificity, which has not been described previously. However, the roles of these MRPs in the development of MS and its cardiac complications still need further study.

In conclusion, this study is the first to compare the mitochondrial genome encoded proteins expression in different tissues of the diet induced metabolic syndrome rat model. Our results showed that the mitochondrial morphologic change and the protein expression of the myocardiocytes were different from those of hepatocyte and skeletal muscle cells. To further clarify the underlying mechanisms, we screened four highly expressed MRPs, MRPS23, MRPL27, MRPL45 and MRPL48 in the cardiomyocytes. Even though their specific roles in the mitochondrial genome encoded proteins expression and development of heart disease are still not very clear, our present study suggested that the apparent tissue specificity of mitochondrial genome encoded proteins expression partially explain why heart is usually more severely affected in MS, which might be one of the underlying mechanisms of heart disease of MS. 


\section{Cellular Physiology Cell Physiol Biochem 2017;43:959-968

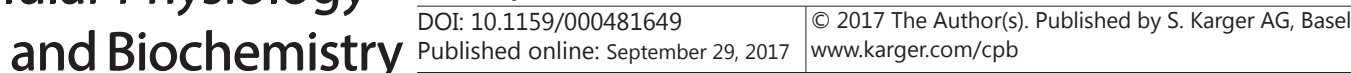 \\ Zheng et al.: The Mitochondrial Mechanisms of the Heart Disease in Metabolic Syndrome}

\section{Acknowledgements}

This work was supported by a grant from National Natural Science Foundation of China (30900567).

\section{Disclosure Statement}

None of the authors has any conflicts of interest with regards to this research.

\section{References}

1 Ford ES, Li C, Zhao G: Prevalence and correlates of metabolic syndrome based on a harmonious definition among adults in the US. J Diabetes 2010;2:180-193.

-2 Sumner AD, Sardi GL, Reed JF, 3rd: Components of the metabolic syndrome differ between young and old adults in the US population. J Clin Hypertens (Greenwich) 2012;14:502-506.

-3 Yang W, Lu J, Weng J, Jia W, Ji L, Xiao J, Shan Z, Liu J, Tian H, Ji Q, Zhu D, Ge J, Lin L, Chen L, Guo X, Zhao Z, Li Q, Zhou Z, Shan G, He J: Prevalence of diabetes among men and women in China. N Engl J Med 2010;362:1090-1101.

4 Executive Summary of The Third Report of The National Cholesterol Education Program (NCEP) Expert Panel on Detection, Evaluation, And Treatment of High Blood Cholesterol In Adults (Adult Treatment Panel III). Jama 2001;285:2486-2497.

5 Alberti KG, Zimmet P, Shaw J: The metabolic syndrome--a new worldwide definition. Lancet 2005;366:1059-1062.

6 Thyfault JP, Krogh-Madsen R: Metabolic disruptions induced by reduced ambulatory activity in free-living humans. J Appl Physiol (1985) 2011;111:1218-1224.

-7 Raza H, John A, Howarth FC: Increased oxidative stress and mitochondrial dysfunction in zucker diabetic rat liver and brain. Cell Physiol Biochem 2015;35:1241-1251.

8 Aral C, Akkiprik M, Caglayan S, Atabey Z, Ozisik G, Bekiroglu N, Ozer A: Investigation of relationship of the mitochondrial DNA $16189 \mathrm{~T}>\mathrm{C}$ polymorphism with metabolic syndrome and its associated clinical parameters in Turkish patients. Hormones (Athens) 2011;10:298-303.

-9 Palmieri VO, De Rasmo D, Signorile A, Sardanelli AM, Grattagliano I, Minerva F, Cardinale G, Portincasa P, Papa S, Palasciano G: T16189C mitochondrial DNA variant is associated with metabolic syndrome in Caucasian subjects. Nutrition 2011;27:773-777.

10 Juo SH, Lu MY, Bai RK, Liao YC, Trieu RB, Yu ML, Wong LJ: A common mitochondrial polymorphism $10398 \mathrm{~A}>\mathrm{G}$ is associated metabolic syndrome in a Chinese population. Mitochondrion 2010;10:294-299.

11 Phung TL, Du W, Xue Q Ayyaswamy S, Gerald D, Antonello Z, Nhek S, Perruzzi CA, Acevedo I, RamannaValmiki R, Rodriguez-Waitkus P, Enayati L, Hochman ML, Lev D, Geeganage S, Benjamin LE: Akt1 and akt3 exert opposing roles in the regulation of vascular tumor growth. Cancer Res 2015;75:40-50.

12 Zheng N, Ding X, Sun A, Jahan R: PDK1 Activity Regulates Proliferation, Invasion and Growth of Hemangiomas. Cell Physiol Biochem 2015;36:1903-1910.

13 Walder K, Oakes N, Fahey RP, Cooney G, Zimmet PZ, Collier GR: Profile of dyslipidemia in Psammomys obesus, an animal model of the metabolic syndrome. Endocr Regul 2002;36:1-8.

14 Galloway CA, Yoon Y: Mitochondrial morphology in metabolic diseases. Antioxid Redox Signal 2013;19:415430.

15 Ilkun 0, Boudina S: Cardiac dysfunction and oxidative stress in the metabolic syndrome: an update on antioxidant therapies. Curr Pharm Des 2013;19:4806-4817.

16 Detmer SA, Chan DC: Functions and dysfunctions of mitochondrial dynamics. Nat Rev Mol Cell Biol 2007;8:870-879.

17 Pich S, Bach D, Briones P, Liesa M, Camps M, Testar X, Palacin M, Zorzano A: The Charcot-Marie-Tooth type 2A gene product, Mfn2, up-regulates fuel oxidation through expression of OXPHOS system. Hum Mol Genet 2005;14:1405-1415.

18 Heinonen S, Buzkova J, Muniandy M, Kaksonen R, Ollikainen M, Ismail K, Hakkarainen A, Lundbom J, Lundbom N, Vuolteenaho K, Moilanen E, Kaprio J, Rissanen A, Suomalainen A, Pietilainen KH: Impaired Mitochondrial Biogenesis in Adipose Tissue in Acquired Obesity. Diabetes 2015;64:3135-3145.

-19 Terman A, Gustafsson B, Brunk UT: Mitochondrial damage and intralysosomal degradation in cellular aging. Mol Aspects Med 2006;27:471-482. 


\section{Cellular Physiology Cell Physiol Biochem 2017;43:959-968 and Biochemistry DOI: 10.1159/000481649 2017 Published 2017 The Author(s). Published by S. Karger AG, Basel

20 Mandavia CH, Pulakat L, DeMarco V, Sowers JR: Over-nutrition and metabolic cardiomyopathy. Metabolism 2012;61:1205-1210.

-21 Acin-Perez R, Fernandez-Silva P, Peleato ML, Perez-Martos A, Enriquez JA: Respiratory active mitochondrial supercomplexes. Mol Cell 2008;32:529-539.

-22 Kim JJ, Battaile KP: Burning fat: the structural basis of fatty acid beta-oxidation. Curr Opin Struct Biol 2002;12:721-728.

23 Mackenzie J, Pedersen L, Arent S, Henriksen A: Controlling electron transfer in Acyl-CoA oxidases and dehydrogenases: a structural view. J Biol Chem 2006;281:31012-31020.

-24 Ruzicka FJ, Beinert H: A new iron-sulfur flavoprotein of the respiratory chain. A component of the fatty acid beta oxidation pathway. J Biol Chem 1977;252:8440-8445.

-25 Ramsay RR, Steenkamp DJ, Husain M: Reactions of electron-transfer flavoprotein and electron-transfer flavoprotein: ubiquinone oxidoreductase. Biochem J 1987;241:883-892.

-26 Beckmann JD, Frerman FE: Electron-transfer flavoprotein-ubiquinone oxidoreductase from pig liver: purification and molecular, redox, and catalytic properties. Biochemistry 1985;24:3913-3921.

-27 Chen Q Vazquez EJ, Moghaddas S, Hoppel CL, Lesnefsky EJ: Production of reactive oxygen species by mitochondria: central role of complex III. J Biol Chem 2003;278:36027-36031.

28 St-Pierre J, Buckingham JA, Roebuck SJ, Brand MD: Topology of superoxide production from different sites in the mitochondrial electron transport chain. J Biol Chem 2002;277:44784-44790.

29 Schonfeld P, Wojtczak L: Fatty acids decrease mitochondrial generation of reactive oxygen species at the reverse electron transport but increase it at the forward transport. Biochim Biophys Acta 2007;1767:10321040.

-30 Cocco T, Di Paola M, Papa S, Lorusso M: Arachidonic acid interaction with the mitochondrial electron transport chain promotes reactive oxygen species generation. Free Radic Biol Med 1999;27:51-59.

31 Schonfeld P, Reiser G: Rotenone-like action of the branched-chain phytanic acid induces oxidative stress in mitochondria. J Biol Chem 2006;281:7136-7142.

-32 Roul D, Recchia FA: Metabolic alterations induce oxidative stress in diabetic and failing hearts: different pathways, same outcome. Antioxid Redox Signal 2015;22:1502-1514.

33 Mattiazzi M, Vijayvergiya C, Gajewski CD, DeVivo DC, Lenaz G, Wiedmann M, Manfredi G: The mtDNA T8993G (NARP) mutation results in an impairment of oxidative phosphorylation that can be improved by antioxidants. Hum Mol Genet 2004;13:869-879.

34 Sgarbi G, Baracca A, Lenaz G, Valentino LM, Carelli V, Solaini G: Inefficient coupling between proton transport and ATP synthesis may be the pathogenic mechanism for NARP and Leigh syndrome resulting from the T8993G mutation in mtDNA. Biochem J 2006;395:493-500.

-35 Tatuch Y, Robinson BH: The mitochondrial DNA mutation at 8993 associated with NARP slows the rate of ATP synthesis in isolated lymphoblast mitochondria. Biochem Biophys Res Commun 1993;192:124-128.

-36 Ye W, Chen S, Jin S, Lu J: A novel heteroplasmic mitochondrial DNA mutation, A8890G, in a patient with juvenileonset metabolic syndrome: a case report. Mol Med Rep 2013;8:1060-1066.

-37 Kwasniak M, Majewski P, Skibior R, Adamowicz A, Czarna M, Sliwinska E, Janska H: Silencing of the nuclear RPS10 gene encoding mitochondrial ribosomal protein alters translation in arabidopsis mitochondria. Plant Cell 2013;25:1855-1867.

-38 Fung S, Nishimura T, Sasarman F, Shoubridge EA: The conserved interaction of C7orf30 with MRPL14 promotes biogenesis of the mitochondrial large ribosomal subunit and mitochondrial translation. Mol Biol Cell 2013;24:184-193.

-39 Nichols TW, Jr: Mitochondria of mice and men: moderate magnetic fields in obesity and fatty liver. Med Hypotheses 2012;79:287-293.

40 Lemasters JJ, Qian T, He L, Kim JS, Elmore SP, Cascio WE, Brenner DA: Role of mitochondrial inner membrane permeabilization in necrotic cell death, apoptosis, and autophagy. Antioxid Redox Signal 2002;4:769-781.

-41 Lim SK, Gopalan G: Aurora-A kinase interacting protein 1 (AURKAIP1) promotes Aurora-A degradation through an alternative ubiquitin-independent pathway. Biochem J 2007;403:119-127.

42 Harada T, Iwai A, Miyazaki T: Identification of DELE, a novel DAP3-binding protein which is crucial for death receptor-mediated apoptosis induction. Apoptosis 2010;15:1247-1255.

43 Gatza ML, Silva GO, Parker JS, Fan C, Perou CM: An integrated genomics approach identifies drivers of proliferation in luminal-subtype human breast cancer. Nat Genet 2014;46:1051-1059.

-44 Suh I, Weng J, Fernandez-Ranvier G, Shen WT, Duh QY, Clark OH, Kebebew E: Antineoplastic effects of decitabine, an inhibitor of DNA promoter methylation, in adrenocortical carcinoma cells. Arch Surg 2010;145:226-232. 